05,13

\title{
Синтез, структура и магнитные свойства многослойных наногетероструктур FelMgOICrIMgOIFe
}

\author{
(C) E.M. Якунина ${ }^{1}$, E.A. Кравцов ${ }^{1,2}$, Yu.N. Khaydukov ${ }^{3,4}$, H.O. Антропов ${ }^{1,2}$, B.B. Проглядо ${ }^{1}$ \\ ${ }^{1}$ Институт фозики металлов УрО РАН, \\ Екатеринбург, Россия \\ ${ }^{2}$ Уральский фредеральный университет, \\ Екатеринбург, Россия \\ ${ }^{3}$ Институт исследований твердого тела им. Макса Планка, \\ Heisenbergstraße 1, Штутгарт, 70569, Германия \\ ${ }^{4}$ Общество научных исследований им. Макса Планка на ядерном реакторе FRM-II, \\ Lichtenbergstraße 1, Гархинг-бай-Мюнхен, 85748, Германия \\ E-mail: eyakuninaart@gmail.com
}

Поступила в Редакцию 9 апреля 2021 г.

В окончательной редакции 9 апреля 2021 г.

Принята к публикации 19 апреля 2021 г.

\begin{abstract}
Слоистые наногетероструктуры $\mathrm{Fe}|\mathrm{MgO}| \mathrm{Cr}|\mathrm{MgO}| \mathrm{Fe}$ - искусственный ферромагнитный материал, в котором обменное взаимодействие магнитных моментов слоев Fe через промежуточные диэлектрические и металлические слои может приводить к магнитным конфигурациям, не реализующимся в хорошо изученных системах $\mathrm{Fe}|\mathrm{MgO}| \mathrm{Fe}$ и $\mathrm{Fe}|\mathrm{Cr}| \mathrm{Fe}$. В настоящей работе исследованы корреляции структурных и магнитных свойств слоистых наногетероструктур $\mathrm{Fe}(10 \mathrm{~nm})|\mathrm{MgO}(1.5 \mathrm{~nm})| \mathrm{Cr}(t \mathrm{~nm})|\mathrm{MgO}(1.5 \mathrm{~nm})| \mathrm{Fe}(7 \mathrm{~nm})$ (толщина $t=0.9$ и $1.8 \mathrm{~nm})$. Структурные исследования, выполненные с помощью рентгеновской дифрактометрии и рефлектометрии высокого разрешения, подтвердили формирование текстурированной структуры и выявили ее хорошо определенный слоистый характер с резкими межслойными границами.
\end{abstract}

Ключевые слова: слоистые магнитные наногетероструктуры, межслойное магнитное упорядочение, рефлектометрия поляризованных нейтронов, эффект гигантского туннельного магнитосопротивления.

DOI: $10.21883 /$ FTT.2021.09.51256.21H

\section{1. Введение}

$\mathrm{Fe}|\mathrm{MgO}| \mathrm{Cr}|\mathrm{MgO}| \mathrm{Fe}$ - это новая система, в которой магнитные моменты $\mathrm{Fe}$ связаны между собой через диэлектрические и металлические промежуточные слои. Магнитные и магнитотранспортные свойства магнитных многослойных структур с границами раздела металл-металл и металл-диэлектрик были глубоко изучены; напротив, системы, включающие как металлические, так и диэлектрические промежуточные слои, изучены гораздо меныше. В частности, физически интересные системы $\mathrm{Fe}|\mathrm{MgO}| \mathrm{Cr}$ изучены в очень ограниченном количестве публикаций $[1,2]$.

В данной работе мы сообщаем о синтезе, структуре и магнитных свойствах наногетероструктур $\mathrm{Fe}|\mathrm{MgO}| \mathrm{Cr}$. В основном мы сосредоточились на технологии изготовления образцов и их магнитных свойствах, включая макроскопические свойства, а также на получении профилей намагничивания с разрешением на уровне монослоев. Мы исследовали два образца $\mathrm{Fe}(10 \mathrm{~nm})$ $\mathrm{MgO}(1.5 \mathrm{~nm})|\mathrm{Cr}(\mathrm{t} \mathrm{nm})| \mathrm{MgO}(1.5 \mathrm{~nm})|\mathrm{Fe}(7 \mathrm{~nm})| \mathrm{Ta}(5 \mathrm{~nm}) \quad \mathrm{c}$ толщиной $t=0.9 \mathrm{~nm}$ (образец 1) и $t=1.8 \mathrm{~nm}$ (образец 2). Такие толщины $\mathrm{Cr}$, как известно, обеспечивают антиферромагнитное и ферромагнитное упорядочение в сверхрешетках $\mathrm{Fe} \mid \mathrm{Cr}$ [3]. Металлы выращивались методом магнетронного распыления на постоянном токе, слои $\mathrm{MgO}$ - методом высокочастотного распыления. Рентгеновскую рефлектометрию и дифракцию для определения структурных характеристик образцов проводили на дифрактометре Empyrean PANalytical с использованием излучения $\mathrm{CoK}_{\alpha}$ в геометрии параллельного пучка. Намагниченность измеряли методом вибрационной магнитометрии.

\section{2. Эксперимент}

Были исследованы две слоистые наногетероструктуры: $\mathrm{Fe}(10 \mathrm{~nm})|\mathrm{MgO}(1.5 \mathrm{~nm})| \mathrm{Cr}(\mathrm{t})|\mathrm{MgO}(1.5 \mathrm{~nm})| \mathrm{Fe}(7 \mathrm{~nm}) \mid$ $\mathrm{Ta}(5 \mathrm{~nm}), t=0.9 \mathrm{~nm}$ (далее - образец 1) и $t=1.8 \mathrm{~nm}$ (далее - образец 2), выращенные методом высоковакуумного магнетронного напыления на монокристаллические подложки $\mathrm{MgO}$ (100) без какого-либо буферного слоя. Для защиты от окисления готовые структуры покрывались слоем Та. К слоям $\mathrm{MgO}$ в процессе роста была применена специальная процедура отжига. Данная манипуляция была проведена с целью улучшения качества слоистой и кристаллической структуры $\mathrm{MgO}$. Согласно предыдущим исследованиям, отжиг во время роста положительно влияет на формирование кристаллической структуры $\mathrm{MgO}$ [4]. Рентгеноструктурные исследования были выполнены в геометрии параллельного пучка на лабораторном дифрактометре PANalytical 
Empyrean Series 2 с использованием $\mathrm{CoK}_{\alpha}$-излучения. Параллельный пучок сформирован с помощью рентгеновского зеркала $\mathrm{W} \mid \mathrm{Si}$, установленного на первичном пучке, и параллельного пластинчатого коллиматора и плоского графитового монохроматора - на вторичном пучке. Щель для падающего луча составляла $0.05 \mathrm{~mm}$, для дифрагированного луча $-0.1 \mathrm{~mm}$. Измерения рефлектометрии поляризованных нейтронов были выполнены в стандартной $\theta-2 \theta$ геометрии на рефлектометре NREX на исследовательском реакторе FRM II (Гархинг, Германия). Измерения с полным поляризационным анализом проведены на длине волны $4.3 \AA$. Поляризация падающего пучка $P_{\mathrm{p}}>99.99 \%$. Анализ поляризации пучка после рассеяния проводили с помощью анализатора поляризации с эффективностью $P_{\mathrm{a}}=99.1 \%$.

\section{3. Результаты и обсуждение}

Свойства слоистых наносистем и структуры межслойных границ были определены с помощью рентгеновской рефлектометрии и дифракции высокого разрешения. Толщину слоев и среднеквадратичную шероховатость границы раздела определяли методом рефлектометрии. Установлено, что структурные свойства образцов 1 и 2 идентичны, поэтому далее большинство результатов приводится для образца 1. На рис. 1 представлены результаты рефлектометрических исследований наногетероструктур и результаты их обработки (фитирования). Анализ рентгеновских данных проводили с помощью коммерческих программ PANalytical X'Pert Reflectometry и X'Pert Epitaxy. Соответствующие параметры модельной кривой приведены в таблице. Из обработки рефлектометрических кривых установлено, что толщины слоев близки к номинальным, а также наблюдается формирование слоистой структуры с четкими границами раздела во всех слоях, за исключением слоя $\mathrm{Cr}$, у которого наблюдается шероховатость примерно два монослоя. Уменьшенное значение плотности верхнего слоя Fe может быть обусловлено его некоторым структурным несовершенством из-за роста на немонокристаллической прослойке $\mathrm{MgO}$. Плотность нижнего слоя $\mathrm{Fe}$, выращен-

Структурные параметры образца $\mathrm{Fe}(10 \mathrm{~nm})|\mathrm{MgO}(1.5 \mathrm{~nm})|$ $\mathrm{Cr}(0.9 \mathrm{~nm})|\mathrm{MgO}(1.5 \mathrm{~nm})| \mathrm{Fe}(7 \mathrm{~nm})$, полученные после фитинга данных рентгеновской рефлектометрии

\begin{tabular}{c|c|c|c}
\hline $\begin{array}{c}\text { Материал } \\
\text { слоя }\end{array}$ & $\begin{array}{c}\text { Толщина } \\
\text { слоя, } \AA\end{array}$ & $\begin{array}{c}\text { Межслойная } \\
\text { шероховатость, } \AA\end{array}$ & $\begin{array}{c}\text { Плотность, } \\
\mathrm{g} / \mathrm{cm}^{3}\end{array}$ \\
\hline $\mathrm{Fe}$ & $94 \pm 1$ & 0.008 & $9.3 \pm 2$ \\
$\mathrm{MgO}$ & $17 \pm 1$ & 0.02 & $4.2 \pm 2$ \\
$\mathrm{Cr}$ & $5.6 \pm 1$ & 3.1 & $7.8 \pm 2$ \\
$\mathrm{MgO}$ & $24 \pm 1$ & 0.004 & 1.8 \\
$\mathrm{Fe}$ & $63 \pm 0.5$ & 3.3 & $7.4 \pm 3$ \\
$\mathrm{Ta}$ & $44 \pm 0.4$ & 0.002 & $18.4 \pm 7$ \\
$\mathrm{Ta}_{2} \mathrm{O}_{5}$ & $24 \pm 0.3$ & 0.002 & $7.8 \pm 1.5$
\end{tabular}

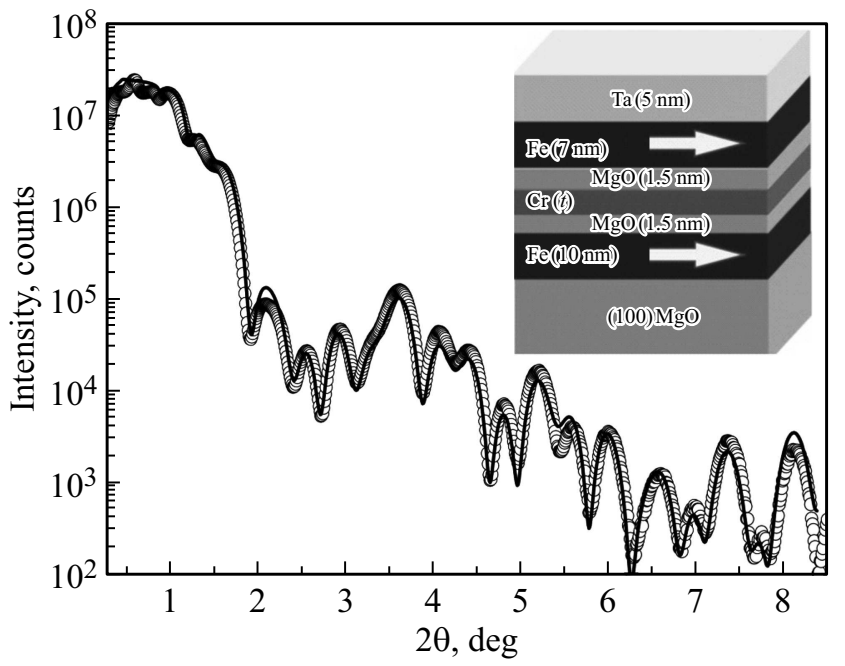

Рис. 1. Экспериментальная рентгеновская рефлектограмма образца 1 (символы) и модельная кривая (линия). На вставке приведена схематическая структура исследуемого образца с параллельным упорядочением магнитных моментов в слоях железа.

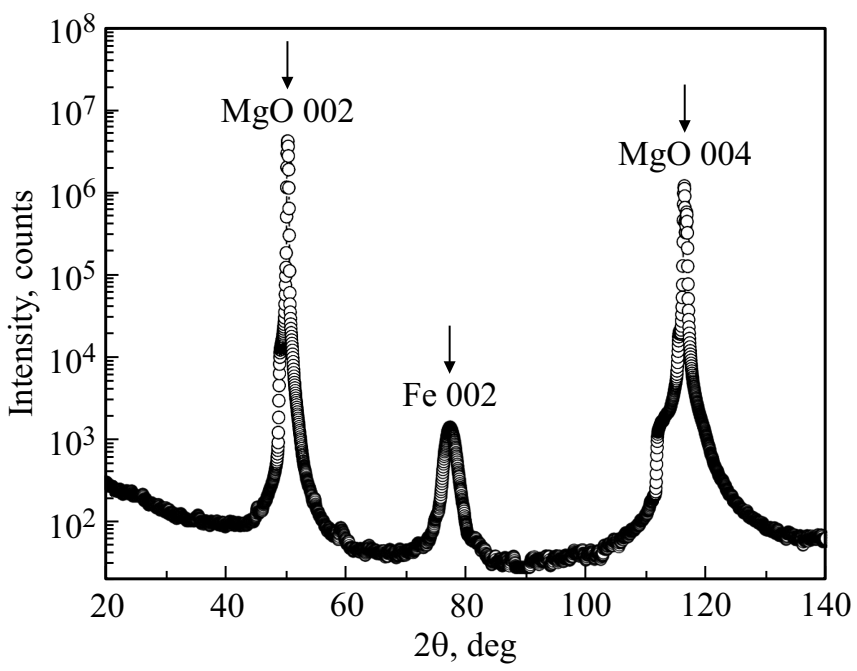

Рис. 2. Рентгеновская дифрактограмма образца 1 с четко определенными структурными пиками, соответствующими брэгговским отражениям $002 \mathrm{MgO}, 004 \mathrm{MgO}$ и $002 \mathrm{Fe}$, свидетельствующая о выраженной текстуре (001) $\mathrm{Fe}$ и (001) $\mathrm{MgO}$ вдоль направления роста образца.

ного на монокристаллической подложке $\mathrm{MgO}$, близка к значению плотности объемного $\mathrm{Fe}$.

Кристаллическая структура образцов была определена с помощью рентгеновской дифракции в геометрии $\theta-2 \theta$ с использованием $\mathrm{CoK}_{\alpha}$-излучения. На рис. 2 представлена дифрактограмма, измеренная для образца 1. Из дифрактограммы видно, что в скане от образца $\mathrm{Fe}(10 \mathrm{~nm})|\mathrm{MgO}(1.5 \mathrm{~nm})| \mathrm{Cr}(0.9 \mathrm{~nm})|\mathrm{MgO}(1.5 \mathrm{~nm})| \mathrm{Fe}(7 \mathrm{~nm})$ присутствуют сигналы, соответствующие брэгговским отражениям (002) $\mathrm{MgO},(004) \mathrm{MgO}$ и (002) Fе. Эти пики свидетельствуют о наличии выраженной текстуры 


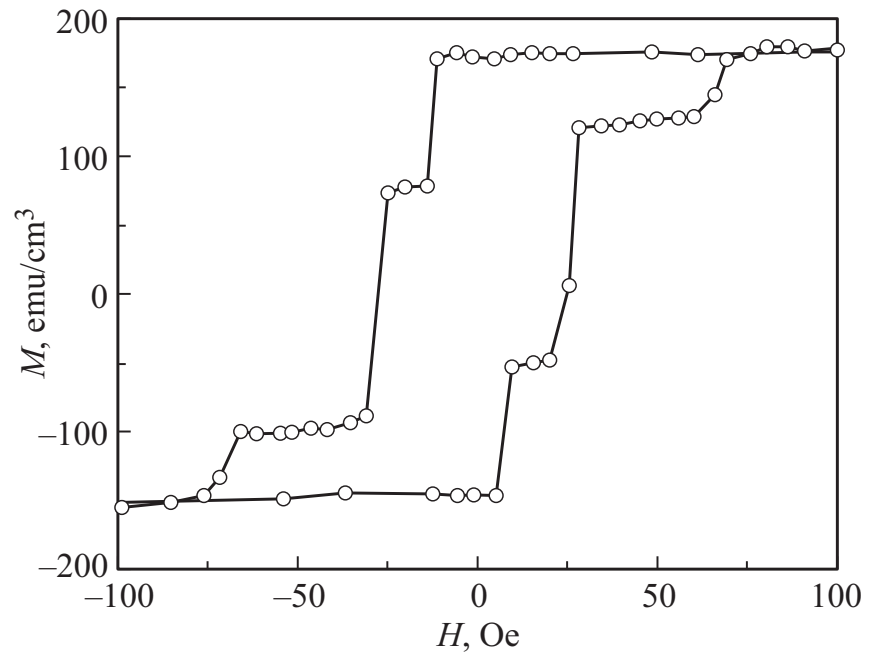

Рис. 3. Петля магнитного гистерезиса образца 1 , измеренная для ориентации внешнего магнитного поля относительно оси легкого намагничивания [100] $\mathrm{Fe}$ при приложении магнитного поля под углом $30^{\circ}$.

(001) $\mathrm{Fe}$ и (001) $\mathrm{MgO}$ в направлении роста образца. Данные рентгеновской дифрактометрии указывают на наличие формирования в исследуемых наногетероструктурах кристаллического $\mathrm{Fe}$ и $\mathrm{MgO}$.

Как видно из приведенного графика на рис. 3, петли гистерезиса имеют особенности, а именно плато около значений магнитного поля $H=15$ и $40 \mathrm{Oe}$. Возможно, в этих полях магнитные моменты слоев Fe направлены вдоль оси легкого намагничивания, и неясно, происходит их когерентное вращение вместе или по отдельности. Такое поведение намагниченности наблюдалось в обоих образцах.

Чтобы получить профили намагниченности с разрешением по глубине слоев при указанных значениях магнитного поля, были проведены эксперименты с поляризованными нейтронами. На рис. 4 и 5 представлены результаты измерений рефлектометрии поляризованных нейтронов для образца 1 , а рис. 6 соответствует аналогичным данным, полученным для образца 2. Измерения были проведены при величинах внешнего магнитного поля $H=15$ и 40 Ое после намагничивания в отрицательном поле $H=-4.5 \mathrm{kOe}$. Во время эксперимента поле прикладывали параллельно направлению [100] подложки $\mathrm{MgO}$. На рис. 4 изображены кривые спин-поляризованного отражения, измеренные при $H=15$ Ое. Кривые отражения без переворота спина $R^{++}$и $R^{--}$- характеризуются полным отражением ниже критического края с $Q_{\text {crit }}=0.16 \mathrm{~nm}^{-1}$ и интерференционными колебаниями выше $Q_{\text {crit. }}$ Кривые разделены, что свидетельствует о наличии в образце одного магнитного момента, который коллинеарен $H$. Отметим, однако, что расщепление кривых при малых значениях $Q$, близких к $Q_{\text {crit }}$, довольно незначительно. В дополнение к кривым отражения без переворота спина в эксперименте наблюдается сильное рассеяние с переворотом спина с пиком при $Q=Q_{\text {crit }}$ с амплитудой порядка 20\%. Наличие такого рассеяния говорит о том, что в системе существует неколлинеарный $H$ магнитный момент. Экспериментальные данные были подогнаны к модельным кривым с использованием известного суперматричного подхода [5]. Некоторое

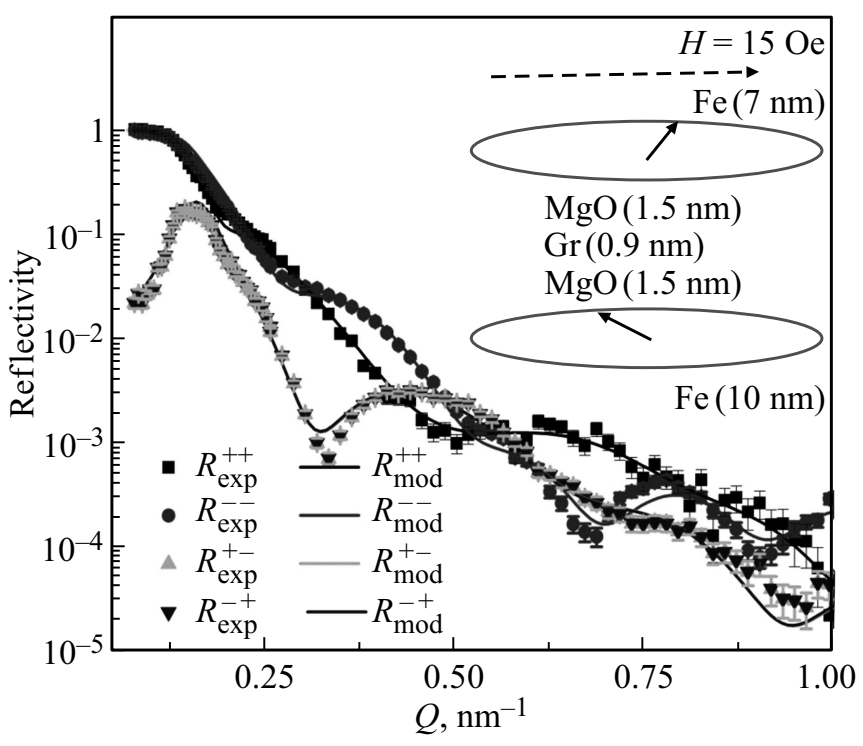

Рис. 4. Экспериментальные кривые рефлектометрии поляризованных нейтронов (символы) и результат подгонки (линии) для образца 1, измеренные в магнитном поле $15 \mathrm{Oе}$. На вставках показано направление магнитных моментов в слоях, определенное в результате подгонки экспериментальных данных к модели.

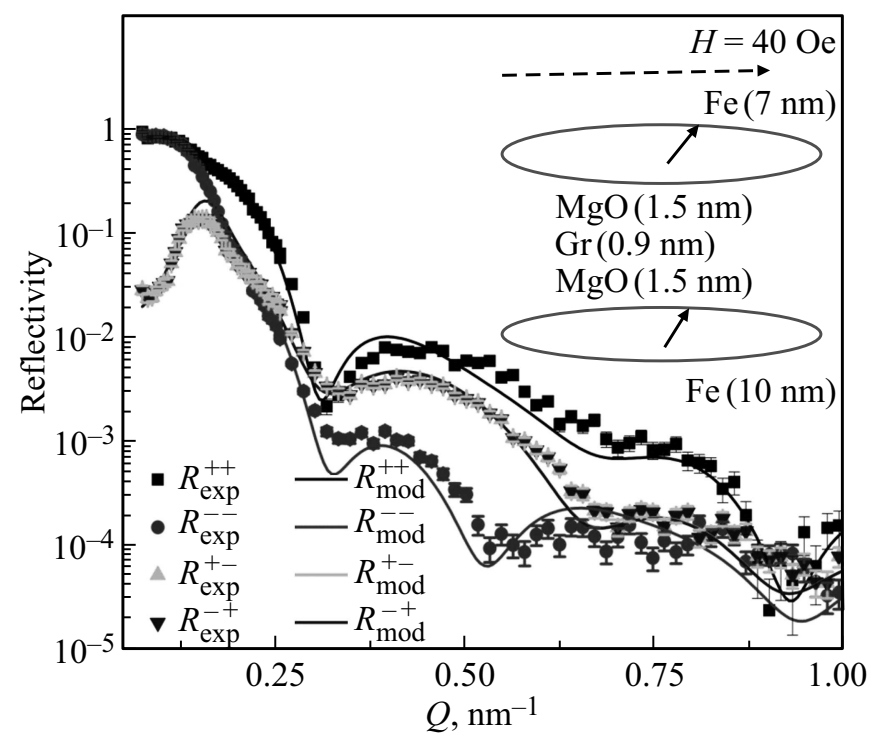

Рис. 5. Экспериментальные кривые рефлектометрии поляризованных нейтронов (символы) и результат подгонки (линии) для образца 1, измеренные в магнитном поле 40 Ое. На вставках показано направление магнитных моментов в слоях, определенное в результате подгонки экспериментальных данных к модели. 


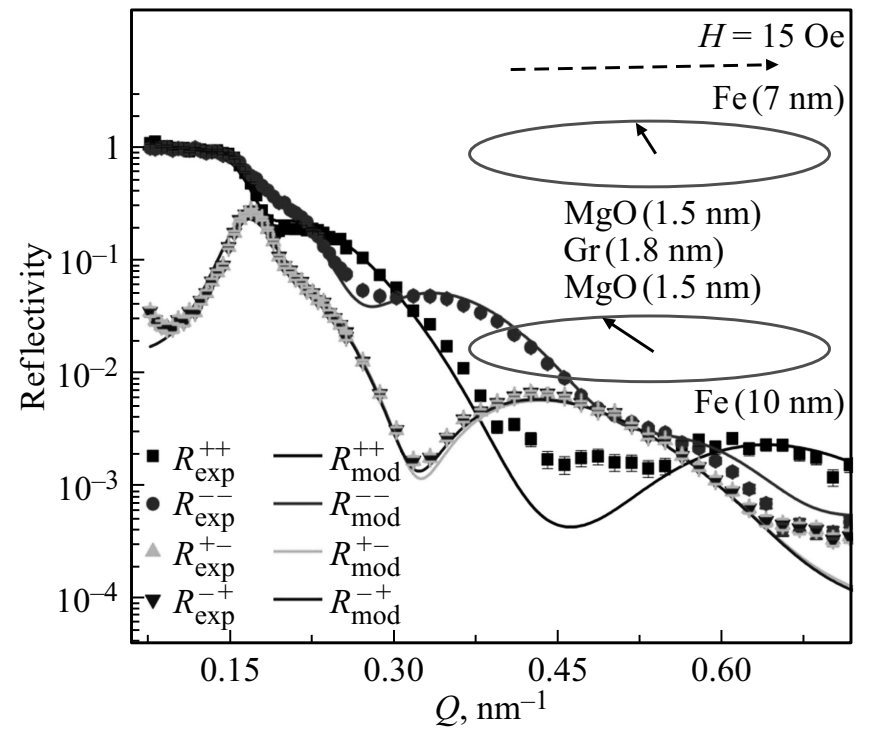

Pис. 6. Экспериментальные кривые рефлектометрии поляризованных нейтронов (символы) и результат подгонки (линии) для образца 2, измеренные в магнитном поле $15 \mathrm{Oe}$. На вставках показано направление магнитных моментов в слоях, определенное в результате подгонки экспериментальных данных к модели.

различие между экспериментальными и теоретическими кривыми на рис. 6 в области малых значений коэффициента отражения объясняется значительным вкладом фона в этой области. При подгонке варьировали намагниченность слоев $\mathrm{Fe}(7 \mathrm{~nm})$ и $\mathrm{Fe}(10 \mathrm{~nm})\left(M_{1}\right.$ и $\left.M_{2}\right)$ и углы между векторами намагниченности и внешним полем $\left(\alpha_{1}\right.$ и $\left.\alpha_{2}\right)$. Наилучшим образом подходят значения $\alpha_{1}=54^{\circ}$ и $\alpha_{2}=154^{\circ}$, чувствительность определения углов около $5^{\circ}$. Соответствующая магнитная конфигурация показана на вставке рис. 4. Видно, что верхний слой железа уже перевернулся и соответствует положительной легкой оси [110], а нижний все еще отвечает отрицательному направлению [110]. Комбинированные магнитные и нейтронографические измерения показали, что коллинеарные компоненты магнитных моментов слоев Fе выровнены и направлены антипараллельно друг другу, так что их сумма близка к нулю [6,7]. Это объясняет относительно небольшую разницу кривых в случае отражения без переворота спина вблизи $Q_{\text {crit. }}$ Эта разница значительно возрастает с увеличением поля до $H=40$ Ое (рис. 5). Однако с увеличением магнитного поля рассеяние с переворотом спина практически не меняется. Количественно это удалось описать, перевернув нижний слой $\mathrm{Fe}$ в направлении положительной легкой оси [110]. Это не меняет неколлинеарный момент, но делает коллинеарные моменты параллельными.

Кривые рефлектометрии поляризованных нейтронов образца 2 при $H=15 \mathrm{Oe}$ (рис. 6) аналогичны кривым образца 1, измеренным в том же поле, и могут быть описаны аналогичным магнитным состоянием. Кроме того, когда поле было увеличено до $H=40$ Ое, кривые отражения без переворота спина вели себя аналогично кривым образца 1: их расщепление увеличилось. Это означает, что коллинеарные компоненты ведут себя в образцах одинаково. Однако наблюдается сильная разница при рассеянии с переворотом спина. Пик сместился от $Q_{\text {crit }}$ к более высокому значению $Q=0.26 \mathrm{~nm}^{-1}$, т. е. неколлинеарная конфигурация образца 2 при $H=40$ Ое отличается от образца 1. С помощью подгонки установлено, что верхний и нижний слои перевернуты в направлениях [1 10$]$ и [110] соответственно. Остальные данные были подогнаны аналогичным образом.

\section{4. Заключение}

С помощью магнетронного напыления мы синтезировали высококачественные слоистые наногетероструктуры $\mathrm{Fe}|\mathrm{MgO}| \mathrm{Cr}|\mathrm{MgO}| \mathrm{Fe}$; их структурные свойства были определены методами рентгеновской рефлектометрии и дифракции. Магнитометрия показывает, что перемагничивание характеризуется наличием промежуточного состояния. Этой неколлинеарностью можно управлять с помощью небольшого внешнего поля в несколько десятков Ое и изменением ориентации образца; также на нее влияет толщина слоя $\mathrm{MgO}$. Были исследованы структурные и магнитные свойства слоистых наногетероструктур $\mathrm{Fe}(10 \mathrm{~nm})|\mathrm{MgO}(1.5 \mathrm{~nm})| \mathrm{Cr}(\mathrm{t} \mathrm{nm})|\mathrm{MgO}(1.5 \mathrm{~nm})|$ $\operatorname{Fe}(7 \mathrm{~nm}) \quad(t=0.9$ и $1.8 \mathrm{~nm})$. Рентгеновские методы подтвердили формирование текстурированной структуры с ориентацией $\mathrm{MgO}[001]\|\mathrm{Fe}[001]\| \mathrm{Cr}[001]$ вдоль нормали к образцу, а также наличие хорошо определенной слоистой структуры с резкими межслойными границами. Проведенное исследование может быть полезно для создания устройств спинтроники для работы при комнатной температуре, а также при низких температурах, в сверхпроводящих спин-вентильных структурах, где требуется неколлинеарность для генерации спинового триплетного конденсата.

\section{Благодарности}

Синтез образцов, магнитометрические и рентгеноструктурные измерения выполнены в центре коллективного пользования ИФМ УрО РАН. Эксперименты по нейтронной рефлектометрии поляризованных нейтронов выполнены на инструменте NREX исследовательского реактора FRM II (Гархинг, Германия).

\section{Финансирование работы}

Исследование выполнено в рамках государственного задания Министерства науки и высшего образования Российской Федерации (тема „Спин“ № AАAА-A18-118020290104-2) при поддержке РФФИ (проект № 19-02-00674). 


\section{Конфликт интересов}

Авторы заявляют, что у них нет конфликта интересов.

\section{Список литературы}

[1] S. Yuasa, D. Djayaprawira. Appl. Phys. 40, 21, 337 (2007).

[2] A. Kozioł-Rachwał, T. Nozaki, V. Zayets, H. Kubota, A. Fukushima, S. Yuasa, Y. Suzuki. J. Appl. Phys. 120, 8, 085303 (2016).

[3] J.A.C. Bland, B. Heinrich (Eds). Ultrathin Magnetic Structures, vol. I-IV. Springer Verlag, Berlin (1994-2005).

[4] Е.М. Якунина, В.И. Боднарчук, В.В. Проглядо, Т.А. Чернышова, Е.А. Кравцов. Поверхность. Рентгеновские, синхротронные и нейтронные исследования 1, 30 (2014).

[5] A. Rühm, B. Toperverg, H. Dosch. Phys. Rev. B 60, 23, 16073 (1999).

[6] E. Młyńczak, K. Freindl, N. Spiridis, J. Korecki. J. Appl. Phys. 113, 2, 024320 (2013).

[7] X. Jia, K. Xia, G.E.W. Bauer. Phys. Rev. Lett. 107, 17, 176603 (2011).

Редактор Е.В. Толстякова 